\title{
Perception and Prevalence of Dietary Supplements among Gym Users in Nasiriyah City: A Cross-sectional Pilot Study in Thi-Qar Provence, Southern Iraq
}

\author{
Farouk M. Sakr ${ }^{1, *}$, Khansaa A. Hussein ${ }^{2}$, Ammar M. Atiyah $^{1}$, Tarig M. Hassan ${ }^{1}$, Al-Saboonchi A. A. ${ }^{1}$ \\ ${ }^{1}$ College of Pharmacy, National University of Science and Technology, Al-Nasiriya, Iraq \\ ${ }^{2}$ College of Dentistry, National University of Science and Technology, Al-Nasiriya, Iraq
}

Received July 26, 2021; Revised August 23, 2021; Accepted October 17, 2021

\section{Cite This Paper in the following Citation Styles}

(a): [1] Farouk M. Sakr, Khansaa A. Hussein, Ammar M. Atiyah, Tarig M. Hassan, Al-Saboonchi A. A. , "Perception and Prevalence of Dietary Supplements among Gym Users in Nasiriyah City: A Cross-sectional Pilot Study in Thi-Qar Provence, Southern Iraq," International Journal of Human Movement and Sports Sciences, Vol. 9, No. 6, pp. 1244 - 1253, 2021. DOI: 10.13189/saj.2021.090619.

(b): Farouk M. Sakr, Khansaa A. Hussein, Ammar M. Atiyah, Tarig M. Hassan, Al-Saboonchi A. A. (2021). Perception and Prevalence of Dietary Supplements among Gym Users in Nasiriyah City: A Cross-sectional Pilot Study in Thi-Qar Provence, Southern Iraq. International Journal of Human Movement and Sports Sciences, 9(6), 1244 - 1253. DOI: 10.13189/saj.2021.090619.

Copyright $\mathrm{C} 2021$ by authors, all rights reserved. Authors agree that this article remains permanently open access under the terms of the Creative Commons Attribution License 4.0 International License

\begin{abstract}
Background: Dietary supplements (DSs) are widely used in gymnastics around the world to improve health, save energy and increase strength. However, consumers should be familiar with the required knowledge regarding the benefits and risks of these supplements. Objective: To assess the perception and prevalence of dietary supplements among Gymnastics users in the city of Nasiriyah. Material and methods: The study was a pilot descriptive cross-sectional survey of 150 Gymnastics both males $(n=75)$ and females $(n=75)$. The data were statistically analyzed and a "p value $\leq 0.05$ " was taken as statistically significant. Results: Participants Socio-demographic characteristics varied widely and were shown to be related to DSs information sources, purpose of use and beliefs. The coaches were the main source of information for males and the internet for females. Males generally use DSs for Bodybuilding, while females mostly use DSs for weight loss. Gymnastics consumed between 1-2 types of DSs with less consumption of three or more. Electrolyte drink, amino acids, vitamin D, calcium, Red Bull or similar, Omega-3, and slimming products were among the most common DSs. Weight gain, ephedra, and anabolic hormones were less often used. Conclusion: The majority of dietary supplement information was received from unreliable sources. The purpose of using DSs differed
\end{abstract}

greatly between males and females and was related to social and demographic factors. The impact of Covid-19 has limited the size of our participants and more research is needed on wider sample of gymnastics after the pandemic to get a better understanding of the use of DSs and their potential impact on health.

Keywords Amino Acids, Nutritional Supplements, Gymnastics, Vitamins, Nutritional Supplements

\section{Introduction}

With the increasing ambition of the Iraqi people to develop a new lifestyle after recovering from the numerous wars and the expulsion of ISIS from the country, the younger generation has shown an interest in their physical abilities and wellness. Individuals interested in being active sports candidates or those wishing to enter military education must pass a physically oriented performance and comply with weight and height standards. The need for rehabilitation and physical exercise as part of healthcare also emphasized the need for gymnastics centres equipped with professional trainers and sports 
doctors. Many Iraqis have realized the importance of improving their new appearance to keep up with the demands of their daily activities. Accordingly, a large number of well-known gyms have been established across the country, as well as health food stores selling all kinds of nutritional supplements.

Dietary supplements (DSs) are neither food nor pharmaceutical products. However, they are intended to supplement individuals with essential nutrients rarely found in regular food [1]. Production and marketing approval for DSs are not required according to US Food and Drug Administration (FDA) regulations [2]. Products are not intended to diagnose, prevent, or treat medical diseases [3] except for vitamin D supplementation to treat deficiency due to rare exposure to sunlight [4].

Nutritional supplements including amino acids, minerals, vitamins, dietary fibre, and fats are defined as dietary supplements, while stimulants, anabolic steroids, pain management, opioids, alcohol, marijuana, and smokeless tobacco are classified as drugs [2].

DSs may have a beneficial effect on the health and performance of individuals. However, the lack of knowledge and awareness of the basic nutritional facts of such supplements makes it easy to persuade or lure users through extensive advertising. Claims on some DSs include safe weight loss, increased energy, improved cognitive and physical performance, pain management, improved general health, and other desirable effects [5-7]. National and international sports authorities have banned hundreds of drugs used among athletes [8]. Athletes have different intentions for using DSs, including bodybuilding, health improvement and performance maintenance [9]. Information about DSs by athletes and gymnasts has been obtained from a variety of sources. Lebanese and Iranian studies have claimed that coaches are the main source of information for DSs among athletes [10, 11].

Potential adverse effects of DSs may include the accumulation of fat-soluble components in the body due to excessive consumption to reach the toxic levels that deplete the liver and kidneys for removal [12]. In addition, the potential adverse interaction of DSs with concomitant foods or medications may result in adverse health risks. Gymnasts in the regular use of these supplements could be at potential risk [12], so that, the lack of information and misuse of DSs can lead to serious health problems. Published studies on herbal supplements found that they were mostly of low quality, did not contain the purported active ingredients and contained unrecognized substances $[13,14]$.

An official investigation reported that a large number of herbal supplements from different sources were analysed and found to contain only about $20 \%$ of the labelled ingredients [15] and some supplements contain heavy metals as well [16]. To our knowledge, rarely reported or no published investigations of DSs use among the Iraqi population have been published in order to provide guidelines for the use and abuse of DSs among athletes in general and gymnasts in particular.

Our investigation was designed as a pilot study due to the impact of Covid-19, with the intention of expanding the study to larger samples of gymnasium users after the pandemic stopped.

\subsection{The Aim of this Study Was Twofold}

\subsubsection{Specific goal}

- To assess the information, prevalence and beliefs and purpose of consuming DSs among male and female gymnasts in Nasiriyah city, Thi-Qar Provence, southern Iraq.

- To relate DSs participants' consumption, information, beliefs, and purpose to their different sociodemographic characteristics.

\subsubsection{Secondary goal}

- To compare our results with similar investigations available

- To determine which gymnast group is most reluctant to take a particular supplement

To make recommendations on the issue.

\section{Materials and Methods}

A descriptive cross-sectional survey was conducted in Arabic and English among recruited gym users from five reputable centres located in Thi-Qar Provence to assess participants' perception and prevalence regarding the consumption of DSs.

\subsection{Study and Sampling Population}

The study was prepared and conducted between October and December 2020. A total of 150 male and female gymnasts divided into two equal groups $(n=75)$.

\subsection{The Inclusion Criteria}

- Gymnasts who have been in regular training

- 18 years and over

- Live in Nasiriyah, Thi-Qar

\subsection{Exclusion Criteria}

\section{- Coaches}

- Professional athletes

\subsection{Study Tools}

A preliminary study showed that a significant number of respondents were DSs consumers. We aimed to recruit 150 participants with an average of 30 participants from each of the five sports clubs. The participants were divided into two equal parts (75 participants each), male 
and female.

\subsection{Ethical Consent}

The study protocol was approved by the Ethics Committee of the National University of Science and Technology, Nasiriyah City, Iraq.

\subsection{Official Approval}

All participants were informed of the objectives of the study and signed formal written consents.

\subsection{Data Analysis}

A pre-tested questionnaire was distributed to participants to collect information on demographic and socioeconomic data, lifestyle, type of supplement, and information sources. Gymnastic believes regarding the use of DSs was presented in eight different statements and subjected to a 5-point Likert scale ranging from Disagree $=1$, Somewhat Disagree $=2$, Neither agree nor disagree $=$ 3 , Somewhat agree $=4$, and agree it $=5$. Data were statistically evaluated in terms of descriptive frequency of ancestry, chi square cross-tab, and Pearson correlations between variables for statistical significance of the data. Statistical analysis was performed using the Statistical Package for Social Studies software (IBM SPSS version 23; USA). All tests were two-sided and a value $\leq 0.05$ was considered statistically significant.

\section{Results}

\subsection{Socio-Demographic Characteristics}

A total of 150 male and female gymnastics participants $(n=75$ each) were included in the current study. Their demographic characteristics are shown in Table 1.

It is clear from the table that the age distribution was significantly different $(p=0.007)$, with the majority being less than 30 years old. Smoking habits differed significantly among the participants as the majority (78.0\%) were non-smokers versus $21.3 \%$ of males while none of the females smoked $(p<0.001)$. Education level varied significantly $(\mathrm{P}<0.001)$ between participants from primary school and below (10.7\%), high school graduates and below (35.3\%, female-dominated), and college students/or graduates $(43.3 \%$, mostly male). and graduate students $(10.7 \%$, estimated by females). The number of DS products consumed showed a significant difference ( $P$ $<0.001)$ between participants in gender groups. The majority consumed one or two products (mostly males) in special proportions $(28.7 \%)$ and $(46.7 \%)$ followed by 3 or $>3$ products $(18.0 \%)$ and $(6.6 \%)$ respectively by females.

Significant differences $(\mathrm{P}<0.001)$ were observed regarding gymnasts' monthly income as the majority (41.3\%) ranged between 1000-2000 dollars followed by 2000-3000 dollars (15.3\%). The lowest income rates were $10.0 \%$ and $8.7 \%$ for those $<500$ and $>3,000$. Dollars.

Table 1. Demographic characteristics of gymnastics users (Total $n=150$, Male $n=75$, Female $n=75$ )

\begin{tabular}{|c|c|c|c|c|}
\hline Demographic Characteristics & $\begin{array}{c}\text { Males } \\
\text { Frequency (\%) }\end{array}$ & $\begin{array}{c}\text { Females } \\
\text { Frequency }(\%)\end{array}$ & $\begin{array}{c}\text { Total } \\
\text { Frequency (\%) }\end{array}$ & $\begin{array}{c}\mathbf{P} \\
\text { Sig. }\end{array}$ \\
\hline Gender & $75(50.0)$ & $75(50.0)$ & $150(100)$ & \\
\hline $\begin{array}{c}\text { Age in Years } \\
18-25 \\
26-30 \\
31-40 \\
40 \text { yeas } \\
\end{array}$ & $\begin{array}{l}38(75.6) \\
25(33.3) \\
12(16.0) \\
0.0(0.0)\end{array}$ & $\begin{array}{l}30(40.0) \\
22(29.3) \\
12(16.0) \\
11(14.6)\end{array}$ & $\begin{array}{c}68(45.0) \\
47(31.3) \\
24(16.0) \\
11(7.4) \\
\end{array}$ & 0.007 \\
\hline $\begin{array}{c}\text { Smoking Habits } \\
\text { Yes } \\
\text { No } \\
\end{array}$ & $\begin{array}{l}32(42.6) \\
43(57.3)\end{array}$ & $\begin{array}{l}0.0(0.0) \\
75(100)\end{array}$ & $\begin{array}{c}32(21.3) \\
118(78.7) \\
\end{array}$ & $<0.001$ \\
\hline $\begin{array}{c}\text { Education Level } \\
\text { Primary schools or below } \\
\text { High school or less } \\
\text { University students / or graduates } \\
\text { Alumni }\end{array}$ & $\begin{array}{c}16(21.3) \\
15(20.0) \\
40(53.3) \\
4(5.3)\end{array}$ & $\begin{array}{l}0.0(0.0) \\
38(50.6) \\
25(33.3) \\
12(16.0)\end{array}$ & $\begin{array}{l}16(10.7) \\
53(35.3) \\
65(43.3) \\
16(10.7)\end{array}$ & $<0.001$ \\
\hline $\begin{array}{c}\text { Number of used DSs } \\
0 \\
1 \\
2 \\
3 \\
3 \\
\end{array}$ & $\begin{array}{c}0.00 \\
27(36.0) \\
48(64.0) \\
0.0(0.0) \\
0.0(0.0)\end{array}$ & $\begin{array}{c}0.00 \\
16(21.3) \\
22(29.3) \\
27(36.0) \\
10(13.3)\end{array}$ & $\begin{array}{c}0.00 \\
43(28.7) \\
70(46.7) \\
27(18.0) \\
10(6.6)\end{array}$ & $<0.001$ \\
\hline $\begin{array}{c}\text { Monthly income USA dollar } \\
<500 \\
500-1000 \\
1000-2000 \\
2000-3000 \\
3000\end{array}$ & $\begin{array}{l}15(20.0) \\
19(25.0) \\
29(38.6) \\
12(16.0) \\
0.0(0.0)\end{array}$ & $\begin{array}{c}0 .(0.0) \\
18(24.0) \\
33(44.0) \\
11(14.6) \\
13(17.3)\end{array}$ & $\begin{array}{c}15(10.0) \\
3724.7) \\
62(41.3) \\
23(15.3) \\
13(8.7)\end{array}$ & $<0.001$ \\
\hline
\end{tabular}




\subsection{Information Source}

The main gymnastics sources of information on DSs are presented in Table 2 .

The difference in the sources was not statistically significant $(p=0.169)$ for the total participants and within the male and female groups. The majority of participants got their information from online advertising $(\mathrm{n}=32$; $21.3 \%)$, coaches $(\mathrm{n}=29 ; 19.3 \%)$, television advertising $(\mathrm{n}$ $=23 ; 15.3 \%)$, heard from other users $(\mathrm{n}=20 ; 13.4 \%)$, more than one source $(\mathrm{n}=19 ; 13.3 \%)$, pharmacists $(\mathrm{n}=$ $16 ; 10.6 \%)$, physicians/dietitians $(\mathrm{n}=11 ; 6.6 \%)$.

\subsection{Purpose of Consuming DS}

Table 3 represents the main purpose of taking the DS and the difference was statistically significant $(\mathrm{p}=0.003)$. Weight loss was the most obvious reason for taking the supplement by most participants $(n=65 ; 43.3 \%$ of the total participants) who were predominantly female $(n=41$; $54.6 \%$ of the female group) followed by these who intend to shape and build their body ( $\mathrm{n}=47,31.3 \%$ of the total) and are mostly males $(\mathrm{n}=31 ; 41.3 \%$ of the male group $)$. Others take DSs to improve their health $(\mathrm{n}=15 ; 10.0 \%)$, to rehabilitate $(\mathrm{n}=10 ; 6.66 \%)$ and least to prevent injury $(\mathrm{n}=2 ; 1.3 \%)$.

\subsection{Gymnasts' Attitudes and Beliefs toward DS}

They are presented in eight 5-points Likert scale statements in Table 4 . The majority of participants agreed significantly $(p<0.05)$ that "DSs make me healthy" $(\mathrm{n}=$ $89 ; 59.3 \%)$, "DSs improve my endurance" $(\mathrm{n}=83$; $55.3 \%)$, "DSs give me energy" ( $\mathrm{n}=73 ; 48.7 \%)$, "DSs increase the amount of training I can do" ( $\mathrm{n}=101 ; 67.3 \%)$, DSs increase my strength ( $\mathrm{n}=67 ; 44.7 \%)$. Most participants including males and females neither agreed nor disagreed with the statement "DSs are safe to use" (n $=54 ; 36.0 \%)$ and the data were not statistically significant $(p=0.155)$. On the other hand, males and females agreed or somewhat agreed with the statement "dietary supplement improved my concentration" at $\mathrm{p}=0.488$ between the two groups.

Table 2. Available sources of knowledge about Dietary supplements (DSs)

\begin{tabular}{|c|c|c|c|}
\hline Information Source & $\begin{array}{c}\text { Males } \\
\text { (OUT of 75) } \\
\text { Frequency (\%) }\end{array}$ & $\begin{array}{c}\text { Females } \\
\text { (OUT of 75) } \\
\text { Frequency (\%) }\end{array}$ & $\begin{array}{c}\text { Total } \\
\text { (OUT of 150) } \\
\text { Frequency (\%) }\end{array}$ \\
\hline Internet advertising & $12(8.0)$ & $20(13.3)$ & $32(21.3)$ \\
\hline Television advertising & $8(5.3)$ & $15(10.0)$ & $23(15.3)$ \\
\hline Heard from other users & $13(10.0)$ & $7(3.3)$ & $20(13.4)$ \\
\hline Trainers & $17(12.7)$ & $12(6.7)$ & $29(19.3)$ \\
\hline Physicians and dietitians & $7(4.0)$ & $4(2.7)$ & $11(6.6)$ \\
\hline Pharmacists & $10(7.3)$ & $6(3.3)$ & $16(10.6)$ \\
\hline More than one source & $8(4.6)$ & $(8.6)$ & $19(13.3)$ \\
\hline
\end{tabular}

Table 3. Purpose of Dietary supplement for male and female gymnastics users

\begin{tabular}{|c|c|c|c|c|}
\hline Purpose & $\begin{array}{c}\text { Male } \\
\text { (OUT of 75) } \\
\text { Frequency }(\%)\end{array}$ & $\begin{array}{c}\text { Female } \\
\text { (OUT of 75) } \\
\text { Frequency (\%) }\end{array}$ & $\begin{array}{c}\text { Total } \\
\text { (OUT of 150) } \\
\text { Frequency (\%) } \\
\end{array}$ & $\begin{array}{c}\mathbf{P} \\
\text { Sig. }\end{array}$ \\
\hline Weight Loss & $24(32.0)$ & $41(54.6)$ & $65(43.3)$ & \multirow{6}{*}{0.00} \\
\hline Shaping and body building & $31(41.3)$ & $16(21.3)$ & $47(31.3)$ & \\
\hline Improve Athletic Performance & $6(4.00)$ & $4(2.66)$ & $10(6.66)$ & \\
\hline Improve Health & $4(2.66)$ & $11(14.66)$ & $15(10.0)$ & \\
\hline Prevent injury & $2(1.33)$ & $0(0.0)$ & $2(1.3)$ & \\
\hline Rehabilitation & $(5.33)$ & $(2.66)$ & $10(6.66)$ & \\
\hline
\end{tabular}


A Cross-sectional Pilot Study in Thi-Qar Provence, Southern Iraq

Table 4. Participants' beliefs and attitudes toward nutritional supplements

\begin{tabular}{|c|c|c|c|c|c|c|}
\hline \multirow[b]{2}{*}{ Statements } & \multicolumn{6}{|c|}{ Frequency (\%) } \\
\hline & Disagree & $\begin{array}{c}\begin{array}{c}\text { Somewhat } \\
\text { disagree }\end{array} \\
\end{array}$ & $\begin{array}{c}\begin{array}{c}\text { Neither agree } \\
\text { nor disagree }\end{array} \\
\end{array}$ & $\begin{array}{c}\text { Somewhat } \\
\text { agree }\end{array}$ & Agree & $\begin{array}{c}\mathbf{P} \\
\text { Sig. }\end{array}$ \\
\hline $\begin{array}{l}\text { 1. Dietary supplements make me healthy } \\
\text { Total }(150) \\
\text { Males }(75) \\
\text { Females }(75)\end{array}$ & $\begin{array}{c}11(7.3) \\
8(10.6) \\
3(4.0)\end{array}$ & $\begin{array}{c}12(8.0) \\
3(4.0) \\
9(12.0)\end{array}$ & $\begin{array}{l}10(6.6) \\
8(10.6) \\
2(2.6)\end{array}$ & $\begin{array}{c}28(18.6) \\
8(10.6) \\
20(26.6)\end{array}$ & $\begin{array}{l}89(59.3) \\
48(64.0) \\
41(54.6)\end{array}$ & 0.006 \\
\hline $\begin{array}{l}\text { 2. Dietary supplements improve my } \\
\text { endurance } \\
\text { Total }(150) \\
\text { Males (75) } \\
\text { Females (75) } \\
\end{array}$ & $\begin{array}{l}0.0(0.0) \\
0.0(0.0) \\
0.0(0.0)\end{array}$ & $\begin{array}{c}6(4.0) \\
6(8.0) \\
0.0(0.0)\end{array}$ & $\begin{array}{c}10(6.6) \\
10(13.3) \\
0.0(0.0)\end{array}$ & $\begin{array}{l}51(34.0) \\
14(18.6) \\
37(49.3)\end{array}$ & $\begin{array}{l}83(55.3) \\
45(60.0) \\
38(50.6)\end{array}$ & $<0.001$ \\
\hline $\begin{array}{l}\text { 3. Dietary supplements are safe to use } \\
\text { Total }(150) \\
\text { Males }(75) \\
\text { Females }(75)\end{array}$ & $\begin{array}{c}13(8.7) \\
5(6.6) \\
8(10.6)\end{array}$ & $\begin{array}{c}24(16.0) \\
7(9.3) \\
17(22.6)\end{array}$ & $\begin{array}{l}54(36.0) \\
29(38.6) \\
25(33.3)\end{array}$ & $\begin{array}{c}18(12.0) \\
11(14.6) \\
7(9.3)\end{array}$ & $\begin{array}{l}41(27.3) \\
23(30.6) \\
18(24.0)\end{array}$ & 0.155 \\
\hline $\begin{array}{l}\text { 4. Dietary supplements provide me with } \\
\text { energy } \\
\text { Total }(150) \\
\text { Males (75) } \\
\text { Females (75) } \\
\end{array}$ & $\begin{array}{l}0.0(0.0) \\
0.0(0.0) \\
0.0(0.0)\end{array}$ & $\begin{array}{l}0.0(0.0) \\
0.0(0.0) \\
0.0(0.0)\end{array}$ & $\begin{array}{c}40(26.6) \\
35(46.6) \\
5(6.6)\end{array}$ & $\begin{array}{l}47(31.3) \\
0.0(0.0) \\
37(49.3)\end{array}$ & $\begin{array}{l}73(48.7) \\
40(53.3) \\
33(44.0) \\
\end{array}$ & $<0.001$ \\
\hline $\begin{array}{l}\text { 5. Dietary supplements increase the } \\
\text { amount of training I can undergo } \\
\text { Total }(150) \\
\text { Males (75) } \\
\text { Females (75) }\end{array}$ & $\begin{array}{c}2.0(1.3) \\
0.0(0.0) \\
2(2.6)\end{array}$ & $\begin{array}{c}3.0(2.0) \\
0.0(0.0) \\
3(4.0)\end{array}$ & $\begin{array}{c}14(9.4) \\
0.0(0.0) \\
14(18.6)\end{array}$ & $\begin{array}{c}30(20.0) \\
0.0(0.0) \\
30(40.0)\end{array}$ & $\begin{array}{c}101(67.3) \\
75(100) \\
26(34.6)\end{array}$ & $<0.001$ \\
\hline $\begin{array}{l}\text { 6. Dietary supplements increase my } \\
\text { strength } \\
\text { Total (150) } \\
\text { Males (75) } \\
\text { Females (75) } \\
\end{array}$ & $\begin{array}{l}9.0(6.0) \\
0.0(0.0) \\
9(12.0) \\
\end{array}$ & $\begin{array}{l}0.0(0.0) \\
0.0(0.0) \\
0.0(0.0)\end{array}$ & $\begin{array}{l}13(8.6) \\
0.0(0.0) \\
13(17.3) \\
\end{array}$ & $\begin{array}{l}61(40.7) \\
28(37.3) \\
33(44.0) \\
\end{array}$ & $\begin{array}{l}67(44.7) \\
47(62.6) \\
20(26.6) \\
\end{array}$ & $<0.001$ \\
\hline $\begin{array}{l}\text { 7. Dietary supplements increase my } \\
\text { ability to cope with pain } \\
\text { Total (150) } \\
\text { Males }(75) \\
\text { Females }(75)\end{array}$ & $\begin{array}{c}0.0(0.0) \\
0.00 \\
0.00\end{array}$ & $\begin{array}{c}12(8.0) \\
3(4.0) \\
9(12.0) \\
\end{array}$ & $\begin{array}{l}52(34.6) \\
0.0(0.0) \\
52(69.3)\end{array}$ & $\begin{array}{l}43(28.6) \\
29(38.6) \\
14(18.6)\end{array}$ & $\begin{array}{l}43(28.7) \\
43(57.3) \\
0.0(0.0)\end{array}$ & $<0.001$ \\
\hline $\begin{array}{c}\text { Dietary supplements improve my } \\
\text { concentration } \\
\text { Total }(150) \\
\text { Male }(75) \\
\text { Female }(75)\end{array}$ & $\begin{array}{l}0.0(0.0) \\
0.0(0.0) \\
0.0(0.0)\end{array}$ & $\begin{array}{l}0.0(0.0) \\
0.0(0.0) \\
0.0(0.0)\end{array}$ & $\begin{array}{l}10(6.7) \\
6(8.0) \\
4(5.3)\end{array}$ & $\begin{array}{l}53(35.3) \\
29(38.6) \\
24(32.0)\end{array}$ & $\begin{array}{l}87(58.0) \\
40(53.3) \\
47(62.6)\end{array}$ & 0.488 \\
\hline
\end{tabular}

Table 5 showed that the consumption of energy supplements differed between males and females $(\mathrm{P}<$ 0.001). Red Bull or similar energy drinks were most preferred by males with the highest consumption among participants $(n=65 ; 43.3 \%)$, followed by sports drinks ( $n$ $=49 ; 32.6 \%)$ and healthy desserts $(\mathrm{n}=10 ; 6.7 \%)$ preferred by females. Vitamin D was the most frequently consumed vitamin $(n=68 ; 45.3 \%)$ with the highest incidence for females $(\mathrm{n}=43 ; 28.6 \%)$ compared to $(\mathrm{n}=$ $25 ; 16.6 \%)$ for males $(\mathrm{p}=0.003)$. Multivitamins came in the second place $(\mathrm{n}=35 ; 23.2 \%)$ with no significant difference between males and females $(\mathrm{p}=0.350)$. Vitamin $\mathrm{E}$ was preferred in males $(\mathrm{n}=13 ; 8.6 \%)$ compared to $(n=0.00)$ for females at $p<0.001$. Vitamin $\mathrm{C}$ and $\mathrm{B}$ complex were the two least consumed at rates of $(\mathrm{n}=18 ; 12.0 \%)$ and $(\mathrm{n}=16 ; 10.6 \%)$ and the difference between the sexes was minimal at $p>0.05$. Males showed that they consumed more iron than females $(\mathrm{P}=0.002)$ while females consumed more calcium $(\mathrm{P}=0.007)$. The carbohydrate and electrolyte drink were consumed at a rate $(n=102 ; 67.9 \%)$ among the total participants and the difference in rates for males and females was significant at " $p<0.001$ ". The consumption of fructose syrup was very low and the difference between groups was small ( $p$ $=0.500$ ). One of the most frequently consumed proteins was amino acids $(\mathrm{n}=94 ; 62.6 \%)$ and the variance in consumption was highly significant for males $(p<0.001)$. Among fish oils, participants preferred to use omega 3 (n $=61 ; 39.9 \%)$ followed by omega 3 and 6 together $(\mathrm{n}=56$; $47.6 \%)$ while omega 6 alone was less $(\mathrm{n}=33 ; 21.9 \%)$. 
There is no statistical difference between male and female consumption of any of the fish oils $(\mathrm{p}>0.05)$. Ginkgo biloba was the most commonly consumed herb $(\mathrm{n}=58$; $29.9 \%)$ followed by ginseng $(n=24 ; 15.9 \%)$ and the rates between the sexes were significant $(p<0.001)$. None of the participants reported the use of ephedra herbal products. With regard to the use of Ergogenic lozenges, slimming products were the most frequently consumed (n $=58 ; 38.6 \%)$ and females accounted for the highest rate $(\mathrm{p}$ $<001)$. Caffeine consumption was second $(\mathrm{n}=33 ; 22.0 \%)$ and coenzyme Q 10 was third $(\mathrm{n}=23 ; 15.2 \%)$ followed by anabolic hormones $(n=15 ; 15.0 \%)$. The consumption of energy adjuvants was significantly higher in favour of males $(p<0.001)$ except for coenzyme Q $10(p=0.326)$.

Table 5. Dietary supplements and frequency of use among gymnasts

\begin{tabular}{|c|c|c|c|c|}
\hline Type of supplements & $\begin{array}{c}\text { Males } \\
\text { (out of 75) }\end{array}$ & $\begin{array}{c}\text { Females (out of } \\
75 \text { ) } \\
\end{array}$ & $\begin{array}{c}\begin{array}{c}\text { Total } \\
\text { (out of 150) }\end{array} \\
\end{array}$ & $\begin{array}{c}\mathbf{P} \\
\text { Sig. }\end{array}$ \\
\hline $\begin{array}{ll} & \text { One of the most consumed Energy supplements } \\
\text { - } & \text { Sport drinks } \\
\text { - } & \text { Health candy } \\
\text { Red bull energy drink or similar }\end{array}$ & $\begin{array}{c}22(14.6) \\
0.00 \\
43(28.6) \\
\end{array}$ & $\begin{array}{l}27(18.0) \\
10(6.7) \\
22(14.6) \\
\end{array}$ & $\begin{array}{l}49(32.6) \\
10(6.7) \\
65(43.3) \\
\end{array}$ & $<0.001$ \\
\hline $\begin{array}{ll} & \text { One of the most consumed Vitamins supplements } \\
\text { - } & \text { Vitamin C } \\
\text { - } & \text { Vitamin D } \\
\text { - } & \text { Vitamin E } \\
\text { - } & \text { Vitamin B complex } \\
\text { - } & \text { Multivitamins }\end{array}$ & $\begin{array}{c}9(6.0) \\
25(16.6) \\
13(8.6) \\
8(5.3) \\
16(10.6) \\
\end{array}$ & $\begin{array}{c}9(6.0) \\
43(28.6) \\
0.00 \\
8(5.3) \\
19(12.6) \\
\end{array}$ & $\begin{array}{l}18(12.0) \\
68(45.2) \\
13(8.6) \\
16(10.6) \\
35(23.2) \\
\end{array}$ & $\begin{array}{c}0.599 \\
0.003 \\
<0.001 \\
0.604 \\
0.350 \\
\end{array}$ \\
\hline $\begin{array}{l}\text { One of the most consumed Mineral supplements } \\
\text { - } \quad \text { Iron } \\
\text { - } \quad \text { Calcium }\end{array}$ & $\begin{array}{l}25(16.6) \\
26(17.3)\end{array}$ & $\begin{array}{l}9.0(6.0) \\
42(28.0)\end{array}$ & $\begin{array}{l}34(22.6) \\
68(45.3)\end{array}$ & $\begin{array}{l}0.002 \\
0.007\end{array}$ \\
\hline $\begin{array}{l}\text { One of the most consumed Carbohydrates } \\
\text { - } \quad \text { Carbohydrate-electrolyte beverage } \\
\text { - } \quad \text { Fructose syrup } \\
\end{array}$ & $\begin{array}{c}62(41.3) \\
13(8.6) \\
\end{array}$ & $\begin{array}{c}40(26.6) \\
12(8.0) \\
\end{array}$ & $\begin{array}{l}102(67.9) \\
25(16.6) \\
\end{array}$ & $\begin{array}{c}<0.001 \\
0.500\end{array}$ \\
\hline $\begin{array}{l}\text { One of the most consumed Protein } \\
\text { - } \quad \text { Amino acid } \\
\text { Weight gain products }\end{array}$ & $\begin{array}{c}75(50.0) \\
0.00 \\
\end{array}$ & $\begin{array}{c}19(12.6) \\
0.00\end{array}$ & $\begin{array}{c}94(62.6) \\
0.00 \\
\end{array}$ & $\begin{array}{c}<0.001 \\
----- \\
\end{array}$ \\
\hline $\begin{array}{ll} & \text { One of the most consumed Fish oils } \\
\text { - } & \text { Omega } 3 \\
\text { - } & \text { Omega } 6 \\
\text { - } & \text { Both } \\
\end{array}$ & $\begin{array}{c}32(21.3) \\
13(8.6) \\
31(20.6) \\
\end{array}$ & $\begin{array}{l}28(18.6) \\
20(13.3) \\
27(18.0) \\
\end{array}$ & $\begin{array}{l}61(39.9) \\
33(21.9) \\
56(47.6) \\
\end{array}$ & $\begin{array}{l}0.309 \\
0.118 \\
0.308 \\
\end{array}$ \\
\hline $\begin{array}{ll} & \text { One of the most consumed Herbals } \\
\text { - } & \text { Ephedra } \\
\text { - } & \text { Ginseng } \\
\end{array}$ & $\begin{array}{c}0.00 \\
22(14.6) \\
43(28.6)\end{array}$ & $\begin{array}{c}0.00 \\
2(1.3) \\
2(1.3)\end{array}$ & $\begin{array}{c}0.00 \\
24(15.9) \\
58(29.9)\end{array}$ & $\begin{array}{l}<---- \\
<0.001 \\
<0.001\end{array}$ \\
\hline $\begin{array}{ll} & \text { One of the most consumed Ergogenic aids } \\
\text { - } & \text { Slimming products } \\
\text { - } & \text { Coenzyme Q10 } \\
\text { - } & \text { Caffeine } \\
\end{array}$ & $\begin{array}{c}2(1.3) \\
10(6.6) \\
27(18.0) \\
15(10.0)\end{array}$ & $\begin{array}{c}56(37.3) \\
13(8.6) \\
6.0(4.0) \\
0.00 \\
\end{array}$ & $\begin{array}{l}58(38.6) \\
23(15.2) \\
33(22.0) \\
15(10.0)\end{array}$ & $\begin{array}{c}<0.001 \\
0.326 \\
<0.001 \\
<0.001\end{array}$ \\
\hline
\end{tabular}


Table 6. Persons' relationships between sociodemographic variables, source of information, purpose, and beliefs about dietary supplement consumption

\begin{tabular}{|c|c|c|c|c|c|c|c|c|}
\hline & & Gender & Age & Education & Income & Information & Purpose & Believe \\
\hline \multirow{3}{*}{ Gender } & Pearson Correlation & 1 & $.213^{* *}$ & .138 & $.351^{* *}$ & -.111 & $-.170^{*}$ & $.392^{* *}$ \\
\hline & Sig. (2-tailed) & & .009 & .092 & .000 & .175 & .037 & .000 \\
\hline & $\mathrm{N}$ & 150 & 150 & 150 & 150 & 150 & 150 & 150 \\
\hline \multirow{3}{*}{ Age } & Pearson Correlation & $.213^{* *}$ & 1 & .008 & $.608^{* *}$ & $.536^{* *}$ & -.016 & $.181^{*}$ \\
\hline & Sig. (2-tailed) & .009 & & .927 & .000 & .000 & .849 & .027 \\
\hline & $\mathrm{N}$ & 150 & 150 & 150 & 150 & 150 & 150 & 150 \\
\hline \multirow{3}{*}{ Education } & Pearson Correlation & .138 & .008 & 1 & .105 & -.002 & -.057 & $.360^{* *}$ \\
\hline & Sig. (2-tailed) & .092 & .927 & & .203 & .980 & .485 & .000 \\
\hline & $\mathrm{N}$ & 150 & 150 & 150 & 150 & 150 & 150 & 150 \\
\hline \multirow{3}{*}{ Income } & Pearson Correlation & $.351^{* *}$ & $.608^{* *}$ & .105 & 1 & $.538^{* *}$ & .109 & $.480^{* *}$ \\
\hline & Sig. (2-tailed) & .000 & .000 & .203 & & .000 & .183 & .000 \\
\hline & $\mathrm{N}$ & 150 & 150 & 150 & 150 & 150 & 150 & 150 \\
\hline \multirow{3}{*}{$\begin{array}{c}\text { Dietary } \\
\text { supplements } \\
\text { (DSs) }\end{array}$} & Pearson Correlation & $.452^{* *}$ & $.369^{* *}$ & -.153 & $.304^{* *}$ & .140 & -.035 & $.403^{* *}$ \\
\hline & Sig. (2-tailed) & .000 & .000 & .061 & .000 & .087 & .671 & .000 \\
\hline & $\mathrm{N}$ & 150 & 150 & 150 & 150 & 150 & 150 & 150 \\
\hline
\end{tabular}

\subsection{Association between Sociodemographic Characteristics of Gymnastics and Perception of DSs}

Participant's sociodemographic characteristics (SDC) and corresponding consumption of DSs, source of information, purpose of use, and gymnastics beliefs are presented in terms of Pearsons' correlations in Table 6.

It is shown from the data that age, income, and consumption of DSs have a positive effect. The relationship with gender and relationships was significantly different at $\mathrm{P}<0.009$.

Gender had positive associations with DSs consumption, purpose and belief, with a very large variance ( $p$ 0.001), but a negative relationship with slightly different responses with information sources $(\mathrm{p}=$ 0.175). DS consumption, information sources, and administrative purpose showed significant differences with age $(\mathrm{P}<0.05)$. Education had a largely clear association with DSs beliefs (p, 0.001), although its relationship to information sources, consumption of DSs, and purpose of use was slightly different $(\mathrm{p}>0.05)$. Gymnastics incomes had significantly different responses with respect to consumption of DSs, source of information and belief in DS $(p<0.001)$, but not in terms of purpose of administration $(p=0.183)$. The information gained about gymnasts did not reveal significant changes in their attitudes to DSs consumption and administrative purpose at non-significant p-values of 0.087 and 0.512, respectively, but indicated largely clear differences in gymnasts' beliefs $(\mathrm{p}=0.002)$.

\section{Discussion}

Dietary supplements are highly demanding products within gymnastics worldwide [17]. The improvement in the standard of living and the stability of social life after Iraq's recovery from the numerous wars and the defeat of ISIS, prompts the population to search for ways to take care of health and stamina in general. Young and middle-aged people are opened to all aspects of life including sports issues with the aim of maintaining their well-being, lifestyle and good looks.

Many well-known gyms equipped with sports facilities have been set up to entice people to join by offering discounted fees and providing qualified trainers. In parallel, many health shops have been set up to sell different types of DSs from all over the world. Even though, physical exercise with or without DSs is essential to managing gymnasts' health and activity performance, their incorrect beliefs and lack of information about the essential health benefits and risks of DSs remain a serious health issue that must be raised to gymnastics concerns.

In the current study, all participants (100\%) of the male and female groups were DS users (with a difference in the number and type of supplements used). This rate is much higher compared to $37.8 \%$ and $36.3 \%$ of similar studies $[10,18]$. The vast majority of participants were non-smokers and had alarming numbers of male smokers and non-females, indicating good health habits among females.

The majority of the study population was under 30 years of age, indicating that gym users are predominantly young adults. In terms of education, gymnasts were mainly college and high school graduates, the rest were 
either middle school or lower and postgraduate. This may indicate that college and high school participants are more enthusiastic about Andover, body shape, and athletic performance. The income of the majority seems to be between 1000-2000 USD and above which is very reasonable for gym fees and DSs purchase prices in Thi-Qar Governorate.

All male and female participants were DSs users with rates of $100 \%$ each. This indicates that males and females are equal in belief in DSs. However, there was a significant difference between the numbers consumed of DSs products between male and female groups. The differences in consumption were quite significant and can be attributed to the different needs and purposes required for the use of DSs between the sexes. Our results are in agreement with those of Knapik et al, [19] demonstrating that the prevalence of most DSs was similar for male and female athletes regardless of the purpose of use.

Similarly, the results of a Saudi study showed that $89.4 \%$ of the males and $10.6 \%$ of the females were from Lebanon, where males constituted $72 \%$ of the DS consumers compared to $28 \%$ of the females. In an Iranian study, males represented $86.8 \%$ of the study participants while females constituted $11.2 \%[10,11]$.

Various sources of information about DSs are presented in Table 2. It is clear that information is mostly obtained in descending order from internet, advertisements, from trainers, television advertisements, heard from other users, from more than one source, from pharmacists, doctor, and Nutritionists. The rates of responses to the sources of information between participants, male and female groups was not statistically significant $(p=0.169)$ noting that all males and females received their information from the same sources. By the way, online advertisements and gym trainers have shown that they are the main sources of information. These results are consistent with those of a Lebanese study where trainers and electronic sources were the main sources of information [10]. A study from Iran showed that trainers $(65 \%)$, nutritionists $(30 \%)$ and doctors $(25 \%)$ were the main sources of information [11] while a Saudi report stated that more reliable sources of information were obtained from doctors (45.9\%) and nutritionists (28.5\%) [20], however, doctors and dieticians were the least sources of information in our study because they were not regularly available in many gyms in Thi-Qar Governorate. Gymnastics appears to rely on community pharmacists as a reliable source of information.

The results in Table 3 reflect the purpose of supplementation among gymnasts. Weight loss was the main concern of all participants, especially females. Body shaping and building came next and they were mainly associated with males. Improving health was the third, followed by improving gymnastics performance and recovery, while using DSs to prevent injury was the lowest purpose. Males and females showed statistically significant differences $(P=0.003)$ in their intent to use the DSs in a way that suits both masculine and feminine needs.

Table 4 represents participants' beliefs and attitudes toward the use of their DSs. The majority of participants, including both the male and female groups, highly agreed with the statement "the supplement makes me healthy" which was in agreement with the findings of Ayman et al., [18].

Male and female participants agreed or somewhat agreed with the statements "Dietary supplements have improved stamina", "Dietary supplements give me energy", "Dietary supplements increase the amount of training I can undergo", "Dietary supplements increase my strength", "Supplements increase my ability to handle pain" and the difference in responses was significant between the sex groups. Both the male and female groups agreed or somewhat agreed on the statement "dietary supplements improved my concentration" and their response rates differed significantly $(\mathrm{p}=0.488)$. Participants seemed sceptical about the statement "dietary supplements are safe to use" as they neither agreed nor disagreed about the statement with slight differences in response between the sexes $(p=0.155)$. The current data indicate that all participants believe in DS to some extent.

A variety of DS items are presented in Table 5. Dietary supplements are classified into groups according to their nature and purpose of use. Participants were asked to choose one of the most consumed items in each group. Of the energy supplements, Red Bull or similar scored the highest among participants and within the male group compared to females $(\mathrm{P}<0.001)$. The women's group favoured sports drinks and healthy sweets more and more. Red Bull and the like are a stronger energy supplement compared to other sports drinks and healthy candy, so males may prefer it due to their extended hard workouts. Among the vitamins, vitamin D was the most consumed by all participants, and females were the most in demand compared to males $(\mathrm{P}<0.001)$. The reason could be vitamin D deficiency among the residents of Thi-Qar. Multivitamins ranked the second after vitamin D and could be used as a popular supplement for both males and females with no significant difference in response $(\mathrm{p}=$ 0.350). The vitamin $C$ and $B$ complex was used equally by males and females at $\mathrm{P}=0.599$ and $\mathrm{P}=0.604$, respectively. Vitamin E appears to be preferred by males at a significantly higher rate compared to females $(\mathrm{P}<$ 0.001). Turning to the side of mineral consumption, calcium was the most consumed especially by females followed by iron predominantly by males with p-values of 0.007 and 0.002 . The women's calcium consumption was paralleled by vitamin D to help benefit from calcium. Carbohydrate and electrolyte drinks were the most frequently consumed by male participants $(\mathrm{p}<0.001)$ while fructose drinks were less frequent and nonsignificant for males and females $(p=0.500)$. Amino 
acid administration was significantly higher in male aspects than in females $(\mathrm{P}<0.000)$. However, excessive consumption of branched-chain amino acids (BCAAs) is associated with an increased risk of type 2 diabetes [21-23].

None of the participants admitted to using weight gain products. Among fish oils, omega-3 was the preferred, followed by a mixture of omega- 3 and omega- 6 , while omega- 6 alone was the least and differences were slight between males and females $p>0.05$. Regarding herbal products, Gingko Biloba was the most preferred followed by ginseng especially on the male side $(p=0.000)$. On the other hand, none of the participants used any of the ephedra products as they might be aware of.

The possible side effect is that Ephedra raises individual blood pressure and is reported to be dangerous in hypertensive subjects when taken concomitantly with caffeine [24, 25].

Taking into account the energy generation aid, the slimming product ranked first and was consumed anonymously by females $(\mathrm{p}<0.001)$ followed by caffeine for males $(p<0.001)$ and coenzyme Q10 for both males and females $(p=0.326)$. Anabolic hormones were the least consumed dietary supplement for males $(\mathrm{P}<0.001)$. The difference in DS proves that each participant used the most appropriate supplement they needed for the purpose and results of their training. The data presented in this study are examined with other results for comparison. A Saudi study [18] reported that protein was the most popular supplement followed by amino acids, multivitamins, creatine and omega 3 . The effectiveness of protein supplementation in improving muscle mass has been documented [26], however, excessive use of protein supplements can be dangerous to health Some individuals [27]. Another study from the USA showed that the most preferred nutritional supplement were vitamins/minerals, followed by protein shakes, vitamin C, and vitamin E [28]. The International Olympic Committee (IOC) and the National Athletics Association (NCAA) have legalized the above supplements while banning ephedra products [29].

Table 7 includes Pearson's correlation and degree of associative significance between participants' sociodemographic characteristics, DS consumption, information sources, purpose of use, and cumulative beliefs in supplements. It was evident that gender was significantly associated with participants' DSs consumption, purpose of use, and cumulative belief but was not significantly associated with information sources. Participant age is highly correlated with information sources and service managers believe, but was not significant for the purpose of using the DS. Education was significantly correlated with participants' belief only, while participants' income was significantly correlated with consumption of DSs and source of information and beliefs, but not with purpose of use. The source of the information is not related to the consumption of DS nor the purpose of use.

A significant association was found between management purposes and beliefs, However, it showed little association with consumption of DSs and sources of information. From the above correlations, it can be said that gender, age, and income influence the consumption of dietary supplements. Calculated supplementation is believed to be closely related to the participant's sociodemographic characteristics. The purpose of supplementation is only related to gender, but not to age, education, income, and consumption. This proves that the gymnasts used the most appropriate supplements they needed for the purpose and results of their training.

\section{Limitation and Recommendation}

Without the help of the trainer, some participants found it difficult to understand the written form of the study objective. Moreover, the study was limited to 150 participants as there were not many participants who met the inclusion criteria and due to the spread of covid-19 which prevented more participants from using gyms at the time of the study. More participants were needed to be evaluated for better evaluation.

\section{Conclusion}

The use of DSs among gymnasts in the city of Nasiriyah, Thi-Qar, Provence prevails equally among males and females. The main purposes of consuming DSs are weight loss, bodybuilding, and improving health and performance. The Internet and coaches are the main sources of information. Gymnastics equally believe in DSs and use them for different purposes. Vitamin D and calcium were probably the most preferred drugs due to the high prevalence of vitamin D deficiency among the population of Thi-Qar. None of the participants used ephedra products. Dietitians and physicians should be involved in gym centres to improve the use of DSs and the health risks involved.

\section{REFERENCES}

[1] Regan L., Jaime J., Paige E., Paul R., Johanna T., "Why US Adults Use Dietary Supplements," JAMA Intern Med., vol 173, no 5, pp. 355-361, 2013.

[2] U.S. Food and Drug Administration [Internet] Silver Spring (MD): Chapter VI Claims. In: Guidance for industry: A dietary supplement labeling guide; c2005 [updated 2015 Mar 19; cited 2015 May 20].

[3] Wallace T.C., "Twenty Years of the Dietary Supplement Health and Education Act-How Should Dietary 
Supplements Be Regulated?" The Journal of Nutrition, Vol 145, no 8, pp. 1683-1686, 2015.

[4] Pludowski P., Karczmarewicz E., Bayer M., Carter G., Danuta Chlebna-Sokól D et al, "Practical guidelines for the supplementation of vitamin D and the treatment of deficits in Central Europe - recommended vitamin D intakes in the general population and groups at risk of vitamin D deficiency," Endokrynologia Polska, vol 64, no 4, pp. 319-327, 2013.

[5] Senekal M., Meltzer S., Horne A., Abrey N.C., Papenfus L., van der Merwe S., Temple N.J., "Dietary Supplement Use in Younger and Older Men Exercising at Gyms in Cape Town,” S. Afr. J. Clin. Nutr, pp. 1-8, 2019.

[6] Čaušević D., Ormanovic S., Doder I., Covic N., "Analysis of Dietary Supplements Usage among Recreational Athletes in Canton Sarajevo," Homo Sports, vol 19, pp. 1923, 2017.

[7] Ruano J., Teixeira V.H., "Prevalence of Dietary Supplement Use by Gym Members in Portugal and Associated Factors," J. Int. Soc. Sports Nutr, vol 17, pp. 18,2020 .

[8] Reardon C.L, Creado S., "Drug abuse in athletes," Subst Abuse Rehabil, Vol. 5, pp. 95-105, 2014.

[9] Slater G., Phillips S.M., "Nutrition guidelines for strength sports: Sprinting, weightlifting, throwing events, and bodybuilding," Journal of Sport Sciences, vol Sup 1, pp. S67-S77, 2011

[10] El Khoury D., and Antoine-Jonville S., "Intake of nutritional supplements among people exercising in gyms in Beirut city," Journal of Nutrition and Metabolism, vol. 2012, pp. 12, 2012.

[11] Karimian J., Esfahani P.S., "Supplement consumption in body builder athletes," Journal of Research in Medical Sciences, vol. 16, no. 10, pp. 1347-1353, 2011.

[12] Sánchez O., Miranda M., Guerra-Hernández E., "Prevalence of protein supplements use at gyms," Nutrición Hospitalaria, vol. 26, pp. 1168-1174, 2011.

[13] Tucker J., Fischer T., Upjohn L., et al, "Unapproved Pharmaceutical Ingredients Included in Dietary Supplements Associated with US Food and Drug Administration Warnings," JAMA Netw Open, vol. 1, no. 6, 2018. doi:10.1001/jamanetworkopen.2018.3337.

[14] Newmaster Steven G., Grguric M., Shanmughanandhan, D., Ramalingam S., Ragupathy S., "DNA barcoding detects contamination and substitution in North America herbal products," BMC Medicine, vol. 11, pp. 222, 2013.

[15] Martinez-Sanz JM, Sospedra I, Ortiz CM, et al (2017) Intended or Unintended Doping? A Review of the Presence of Doping Substances in Dietary Supplements Used in Sports, Nutrients; 9(10):1093

[16] Korfali S. I., Mrouh M., Hawi T., "Evaluation of heavy metals content in dietary supplements in Lebanon," Chemistry Central Journal, vol. 7, no. 1, pp. 10, 2013.

[17] Garthe I., Maughan R.J., "Athletes and Supplements: Prevalence and Perspectives," Int. J. Sport Nutr. Exerc. Metab, vol. 28, pp. 126-138, 2018.

[18] Ayman H., Jawadi H., Abulmalik M., et al, "Prevalence of Dietary Supplements Use among Gymnasium Users," Journal of Nutrition and Metabolism, Vol. 2017, pp. 8, 2017.

[19] Knapik J.I., Steelman R.A., Hoedebecke S.S., Austin K.G., et al. "Prevalence of Dietary Supplement Use by Athletes: Systematic Review and Meta-Analysis," Sports Med vol. 46, pp. 103-123, 2016.

[20] Aljaloud S.O., Ibrahim S.A., "Use of dietary supplements among professional athletes in Saudi Arabia," Journal of Nutrition and Metabolism, vol. 2013, pp. 7, 2013.

[21] Zheng Y., Li Y., Qi Q., Hruby A., et al, "Cumulative consumption of branched-chain amino acids and incidence of type 2 diabetes," Int J Epidemiol, vol. 45, no. 5, pp. 1482-1492, 2016.

[22] Ziegenfuss T., Lowery L., Lemon P., “Acute fluid volume changes in men during three days of creatine supplementation," Journal of Exercise Physiology, vol. 1, no. 3, 1998 .

[23] Groeneveld G.J., Beijer C., Veldink J.H., Kalmijn S. J., Wokke J.H., Den Berg L.H.V., "Few adverse effects of long-term creatine supplementation in a placebo-controlled trial," International Journal of Sports Medicine, vol. 26, no. 4, pp. 307-313, 2005.

[24] Berman J.A., Setty A., Steiner M.J., Kaufman K.R., et al, "Complicated hypertension related to the abuse of ephedrine and caffeine," J Addict Dis, vol. 25, no. 3, pp. 45-8, 2006.

[25] Sander G.E., "Drugs that increase blood pressure," Therapy, vol. 8, no. 3, pp. 275-282, 2011.

[26] Kerksick C.M., Wilborn C.D., Roberts M.D., Smith-Ryan A., Kleiner S.M., Jäger R., Collins R., Cooke M., Davis J.N., Galvan E., et al., "ISSN Exercise \& Sports Nutrition Review Update: Research \& Recommendations," J. Int. Soc. Sports Nutr., vol. 15, pp. 38, 2018.

[27] Kårlund A., Gómez-Gallego C., Turpeinen A.M., Palo-oja O.M., El-Nezami H., Kolehmainen M., "Protein Supplements and Their Relation with Nutrition, Microbiota Composition and Health: Is More Protein Always Better for Sportspeople?" Nutrients, vol. 11, pp. 829, 2019.

[28] Jenkinson D.M., Harbert A.J., "Supplements and sports," American Family Physician, vol. 78, no. 9, pp. 1039-1046, 2008.

[29] Patil N., Narendranath S., Ramachandra K., Somashekar H.S., "Drug abuse in sports," Journal of Pharmacy Research, vol. 5, no. 1, pp. 593-603, 2012. 